\title{
Cross-twinning model of fcc crystal growth
}

\author{
Benjamin W. van de Waal * \\ Department of Physics, TN5238, University of Twente, P.O. Box 217, 7500 AE Enschede, The Netherlands
}

Received 1 June 1995; accepted 15 August 1995

\begin{abstract}
The theory developed in 1960 by Wagner, Hamilton and Seidensticker (WHS-theory) to explain observed crystal growth phenomena in Ge is critically reviewed and shown to be capable of explaining preservation of $\mathrm{ABC}$ stacking order in two dimensions in fcc crystals of effectively spherical closed shell molecules. In order to preserve this stacking order in all directions, i.e. to explain isotropic three-dimensional fcc crystal growth, the WHS-model is extended to contain at least two (crossing) twin lamellae, rather than one. The implications for the atomic arrangement in the crossing region are examined, with the main result that local fivefold symmetry is to be expected. This is related to the frequent observation of multiply twinned particles (MTPs), exhibiting fivefold symmetry, of fcc materials whose preference for fcc over hcp cannot be explained satisfactorily. Accordingly, it is proposed to view the atomic arrangement in the crossing region not as a result of cross-twinning, but rather as its origin, i.e. to think of fcc crystal growth as a process starting with the coalescence or intergrowth of decahedra with fivefold symmetry. Experimental evidence in support of this model is given. A possible implication of the proposed growth model, i.e. that the observed crystal structure of a substance is not necessarily that of lowest free energy for the infinite crystal, but rather corresponds to a local minimum that is made accessible by the kinetics of a particular growth process, is discussed.
\end{abstract}

\section{Introduction}

The high-temperature forms of van der Waals (vdW) crystals of effectively spherical molecules, including the heavier rare gases, are cubic close packed (or face centered cubic, fcc), rather than hexagonal close packed (hcp), with only a few exceptions (e.g. $\mathrm{N}_{2}$ ). This is rather surprising, since the usually small difference in binding energy at $0 \mathrm{~K}$ between the two modifications, as calculated with a variety of two- and more-body potentials, would suggest a low stacking fault energy, and, conse-

\footnotetext{
* Corresponding author. Fax: +31 53 4891101; E-mail: vdwaal@utctv6.ct.utwente.nl.
}

quently, a high contribution to the term TS in the Gibbs free energy at finite temperatures, easily outweighing the energy connected with the faults! Thus a completely random stacking sequence of close packed layers, like ...BCABACABA..., would seem to be more favourable than either ...ABCABC... (fcc) or ...ABABAB...(hcp). This is, in fact, what is found in computer simulations of crystallization from the melt in simple (mostly Lennard-Jones) systems: although crystallization is clearly observed, the crys-

\footnotetext{
${ }^{1}$ Conversely, a relatively low count of stacking faults in a macroscopic crystal could (perhaps wrongly) suggest a high stacking fault energy; as is proposed in this paper, the resistance against stacking faults may be kinetic in origin.
} 
tals are invariably fcc/hcp mixtures, with no clear preference for one or the other arrangement.

Crystal structure predictions are usually based on energy calculations on perfect, infinite crystals of assumed symmetry. They may be not conclusive or tend to give systematically the wrong answer, notably in the case of the heavier rare gases, where hcp is predicted, clearly in contradiction with observation [1].

The fact that a crystal grows on its surface, and that each molecule in its interior found its place and orientation when on the surface, i.e. that the crystal structure is closely connected with surface processes, is thus completely ignored in model calculations where no surface is present. Also, the essential role that is known to be played by defects in the growth process, i.e. the kinetics involved in the process of crossing energy barriers is ignored in such analyses.

Finally, the simple fact that each macroscopic crystal started small, as a nucleus of some tens of atoms, could imply that the structure of the macroscopic crystal has its origin in the structure, preferred by the nucleus, which may be quite different. Indeed, theoretical and experimental studies of nanocrystals and clusters in the last decade indicate that noncrystalline structures with fivefold symmetry are preferred up to sizes of $10^{3}-10^{4}$ atoms $[2,3]$. Consequently, the crossing size, i.e. the size at which fcc (or hcp) becomes the more favourable structure, is probably too large to make a massive non-crystalline to (single) crystalline structural transition likely, at least in vacuum.

In a supercooled liquid, a critical nucleus may or may not have essentially the same structure as the macroscopic crystal to which it will eventually develop. In the first case, new atoms to stick on the surface would find a "mould" with structural features on the surface that could inform it where to settle. These structural features should remain on the surface after a period of growth, and, consequently, be present on macroscopic crystals as well. Perfect fcc crystals, bounded by low-energy faces, do not carry this information, however, since the chances to nucleate a new layer on a (111) face in correct or incorrect registry are virtually the same for $\mathrm{vdW}$ crystals. A second problem with the first possibility is that isolated crystal fragments, the size of the critical nucleus, are known to be instable with re- spect to transformations involving fivefold symmetry [4]. Obviously, this problem does not exist in the second case (non-crystalline nucleus). Moreover, modelbuilding, adding atoms one-by-one to a small seed (e.g. a 4-atom tetrahedron) in favourable sites, invariably results in non-crystalline structures, with frequent occurrence of fivefold symmetry, as, e.g., the 7-atom pentagonal bipyramid and the 13-atom icosahedron [5]. However, since further additions according to the same algorithm never result in a (even partially) crystalline structure, it is difficult to understand how a non-crystalline nucleus could be the origin of a macroscopic crystal. Two scenarios seem possible: at some size the growing cluster undergoes a glass-crystal transition, or, alternatively, as will be proposed and made plausible in this paper, a non-crystalline, though well-defined cluster, with local fivefold symmetry, initiates nearly perfect fcc crystal growth, leaving the non-crystalline cluster virtually unchanged in the interior of the crystal.

First we will propose a mechanism that ensures correct nucleation of new (111) layers on a (nearly) perfect crystal. To this end we will, in the next section, revisit the theory of twin-accelerated crystal growth, as forwarded by Wagner [6] and by Hamilton and Seidensticker [7] (WHS-theory), and examne, in Section 3, the implications on an atomic

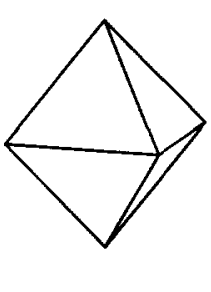

$a$

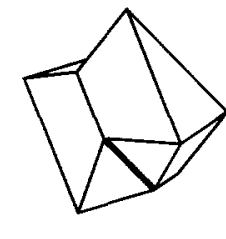

$b$

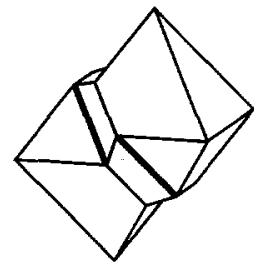

$c$
Fig. 1. The equilibrium shape of many fcc crystals is an octahedron (a), bounded by slow-growing close packed $\{111\}$ faces, exhibiting only threefold coordinated sites of two types, one of which is in incorrect (stacking fault) registry. A simple reflection twin on (111) exhibits grooves (bold lines) and ridges, that alternate around the circumference of the twin plane (b). A second twin plane, parallel to the first, produces grooves where the first twin plane had ridges, and vice versa, resulting in grooves in

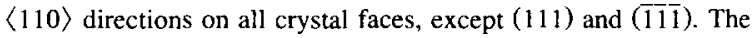
structure (c) can be considered as a single crystal, containing a twin lamella (shaded) parallel to (111). The upper-, middle-, and lower-part of this structure will be referred to as $U, M$ and $L$, respectively, in Figs. 2 and 3. 


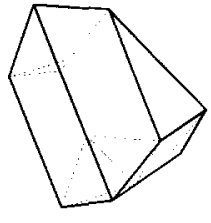

$a$

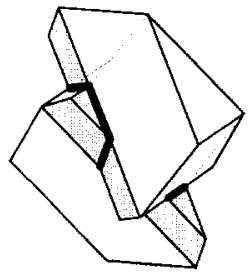

$e$

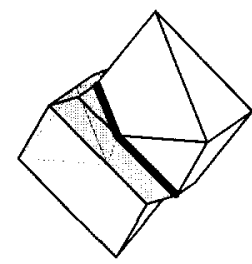

$b$

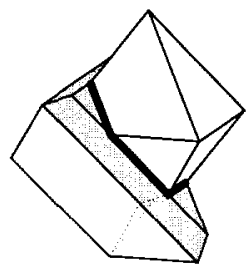

$c d$
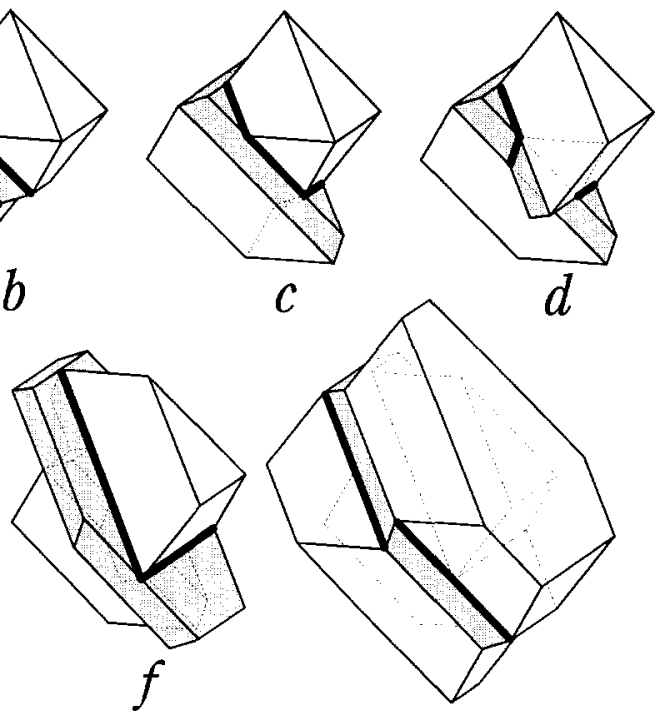

$g$

Fig. 2. Picture summary of WHS-model of twin-accelerated growth. The $141^{\circ}$ grooves in the single twin of Fig. $1 \mathrm{~b}$ offer favourable 4-coordinated sites for surface nucleation. A nucleated island will spread over the two faces forming the reentrant comer, until it reaches the extensions of the adjoining faces. The first layer thus formed exhibits a groove as well, and the process will continue until the situation (a) is reached. There are no grooves left, and the process of rapid growth comes to an end, leaving a triangular platelet (dashed lines mark the original structure). The evolution of the structure of Fig. 1c can be divided in (i) the development of ML, neglecting the presence of $U$ ((b) one reentrant corner filled; (c) all three reentrant comers filled; ML has now essentially the same triangular shape as (a)), and (ii) the development of UM, neglecting the presence of $\mathrm{L}(\mathrm{d})$ one reentrant corner filled; (e) all corners filled). The structure (e) is completely surrounded by a single groove, all reentrant corners being $109.5^{\circ}$, exposing 5 -coordinated sites. Figs. (f) and (g) assume that the $\mathrm{M} / \mathrm{M}$ corners are filled first $(\mathrm{f})$, followed by the $\mathrm{M} / \mathrm{U}$ and $\mathrm{M} / \mathrm{L}$ corners $(\mathrm{g})$. Actually both processes will occur simultaneously. The final structure (g) has the same properties as that of Fig. 1c, i.e. the process will start all over again, and produces thin hexagonal platelets. The representation is highly schematic; thus, the chronological order suggested by the drawing is not real. In fact, as soon as a new groove is created (for example at the start of the process which results in Fig. 2b), nucleation in that groove will take place as well.

scale. A cross-twinning model, which extends the WHS-theory to three dimensions and ensures correct layer deposition in all directions, will be described in Section 4; the atomic arrangement in the crossing region is shown to have fivefold symmetry. The next sections are devoted to experimental evidence and conclusions. This paper is thus essentially concerned with giving kinetic arguments for the observed structure of simple vdW crystals.

\section{The WHS-model}

In 1960 Wagner [6] and, apparently independently, Hamilton and Seidensticker [7], put forward the idea that twinning could be responsible for their observation of essentially two-dimensional growth of fcc germanium crystals, resulting in thin, hexagonal or equilateral triangular platelets with well-developed (111) faces. Here we will briefly review their argument.

Single and doubly twinned crystals, based on an octahedral equilibrium shape, are shown in Fig. $1^{2}$. They exhibit reentrant corners or grooves (bold lines) that may act as sites of preferred surface nucleation. Fig. 2a demonstrates how a single twin will develop into a thin triangular platelet, whereas a double twin spreads in lateral directions (i.e. in the plane of the lamella between the twin planes) to become an

\footnotetext{
${ }^{2}$ Figs. 1 and 2 have been prepared with the program SHAPE V4.1 (1993), Figs. 3-10 with ATOMS V2.3 (1994), both programs by E. Dowty, Shape Software, Kingsport TN.
} 
equally thin but larger hexagonal platelet (Figs. 2b$2 \mathrm{~g}$ ).

Thus, according to this model, and in agreement with many observations, [111] is the direction of slow growth, whereas perpendicular directions, i.e. in the (111) plane, are fast growing directions when (at least) two twin planes parallel to the well developed (111) faces are present. The increased probability for surface nucleation in the grooves, as compared to that on an atomically flat (111) face, results from the fourfold coordination of a site over the groove, as compared to threefold coordination in the two possible (A- and B-) sites on a (111) face. Accordingly, the barrier to nucleation will be lower
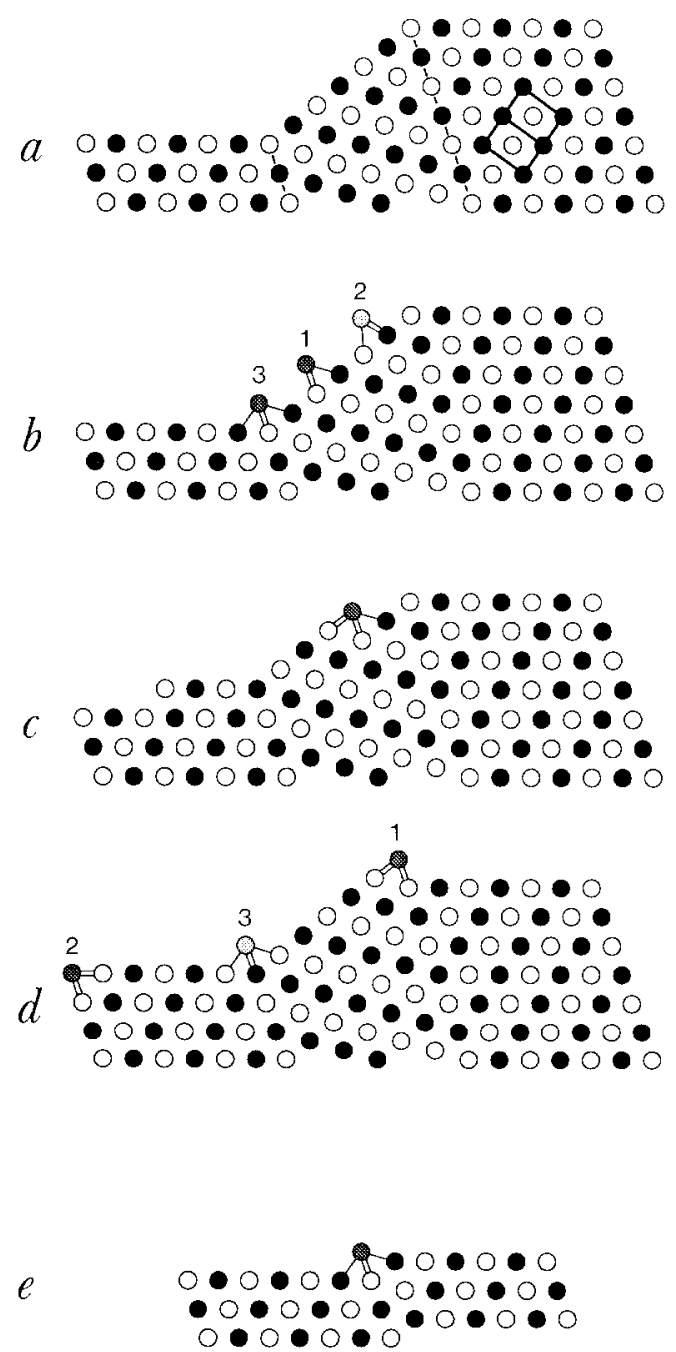

in the groove than on a flat surface, about by a factor 2 , as has been estimated for the special case of two twin planes 1 layer apart [8]. In the following discussion the upper, middle (shaded), and lower part of

Fig. 3. Atomic positions near the (111) surface of an fec crystal containing a single twin lamella $M$ (two closely spaced twin planes - indicated by dashed lines - both parallel to the (111) face). The plane of the drawing $(z=0)$ can be thought of as bisecting the bold line between $\mathrm{M}$ and $\mathrm{L}$ in Fig. $1 \mathrm{c}$ at right angles, cutting the crystal in equal parts. The intersection of the lamella with the (111) surface results in a $141^{\circ}$ groove (or reentrant corner) between parts $L$ and $M$, and a $219^{\circ}$ ridge between parts $M$ and $U$. Circles denote atomic centres: open circles at $z=0, \pm 1$, etc., filled circles at $z= \pm \frac{1}{2}, \pm \frac{3}{2}$, etc., in units of the nn-distance $a / \sqrt{2}$, i.e. the black and white circles mark close packed strings of atoms perpendicular to the page. Shaded circles denote single atoms, or possible atomic positions on the surface: light shading: $z=0$, dark shading $z=\frac{1}{2}$. A single line denotes a single nearest neighbour contact (bond), in the plane of the drawing; double lines denote two bonds, $30^{\circ}$ and $-30^{\circ}$ inclined to the page, respectively. (a) Surface with no adatoms present; the fcc unit cell is outlined. (b) Three possible nucleation sites on the surface: sites 1 and 2 are three-coordinated, and are present everywhere on the surface; site 1 is in correct fcc position, site 2 is in hcp stacking fault position. The reentrant corner exposes 4-coordinated sites (3), where nucleation of a new layer has the higher probability. (c) An atom sitting in site 3 creates new favourable sites (5-coordinated) next to it over the groove (above and below the page), that will stabilize the situation when occupied. A string of atoms over the groove is indistinguishable from an advancing ad-layer, when seen from the L-surface or from the M-surface. When an island has spread by step-flow (Frank-van der Merwe growth) in both directions it is surrounded by favourable 5 -coordinated sites (dark shading), until it reaches the ridge or the end of the crystal. Note that nucleation in a groove prevents stacking disorder. (d) The 5-coordinated sites become 4-coordinated (dark shading), to the effect that there are now three inequivalent, but equally coordinated types of sites on the surface. There is no reason to prefer a type- 3 site over a type- 2 or type- 1 site, so eventually nucleation will take place in all three sites, and the process will repeat itself, until the structure of Fig. $2 b$ is reached, that is: $L$ and $M$ are completed, $U$ is left unchanged. However, the sites next to the atom (row) 1 over the surface of the $\mathrm{U}$-individual have 5 -coordination, and will most probably be occupied as soon as a single atom-row 1 is present. Consequently, the layer that was nucleated in the reentrant comer will spread over the ridge before $\mathbf{L}$ and $\mathrm{M}$ are completed, the ridge being only a minor obstacle. (e) The number of layers in the lamella is immaterial to the process described. The smallest number still having the same properties corresponds to a single ABCBCA stacking fault in the [111] direction. Type- 3 and type-1 sites alternate as soon as they are occupied. The surface exhibits a non-integral surface-step of height $\frac{1}{3} d_{111}$ or $\frac{2}{3} d_{111}$. 
the double twin, Fig. 1c, will be referred to as $U, M$, and $L$, respectively.

\section{WHS-model on an atomic scale}

The atomic scale events are best understood from a hands-on hard-sphere model, but photographs or perspective drawings turn out to be inadequate to bring out the essentials convincingly. Projections that should enable us to discriminate between right ( $\mathrm{fcc}$ ) and wrong (hcp) stacking are preferably made along close-packed rows in close-packed planes, since ABC and ABA stacking can then easily be recognized by straight lines or zig-zag lines, respectively, in the plane of the projection. Thus, a true-to-scale projection of the internal atomic arrangement of the structure of Fig. 1c, along the bold line between parts $\mathrm{M}$ and $\mathrm{L}$ ([101] projection) is shown in Fig. 3. Each of the black and white spheres in the drawing denotes a close-packed row perpendicular to the page $(z=0)$, with each white row having a sphere centre in the plane of the drawing, and each black row having a point of contact between two neighbouring spheres at $z=0$. The black and white rows in the plane of the drawing would also appear as close-packed strings, if the spheres themselves were drawn to scale as well. The main purpose of this picture (full details are given in the legend, which has the form of a self-contained "picture-essay") is to demonstrate that island nucleation in the groove (panels $\mathrm{c}$ and $\mathrm{d}$ ) is always in correct registry, i.e. the grooves are not only sites of preferred nucleation, but also resist wrong stacking. Moreover, the groove is reproduced in each new layer, and thus can act as a "self-perpetuating"' step-source, stimulating uninterrupted $\mathrm{ABCABC}$ stacking.

Each groove must be paid for by an accompanying ridge, that may act as an obstacle, rather than as a stimulus to nucleation and growth. As shown in panel $d$, the ridge can be overcome, however, by nucleation in a fourfold coordinated site, similar to the nucleation in the groove, i.e. preserving the registry and coherence of the layer when it advances over the ridge to the U-part of the crystal. As suggested by Fig. 4, ridges of different types can be overcome in a similar way, only when a defect is present; thus, stepflow around a [101] edge of a

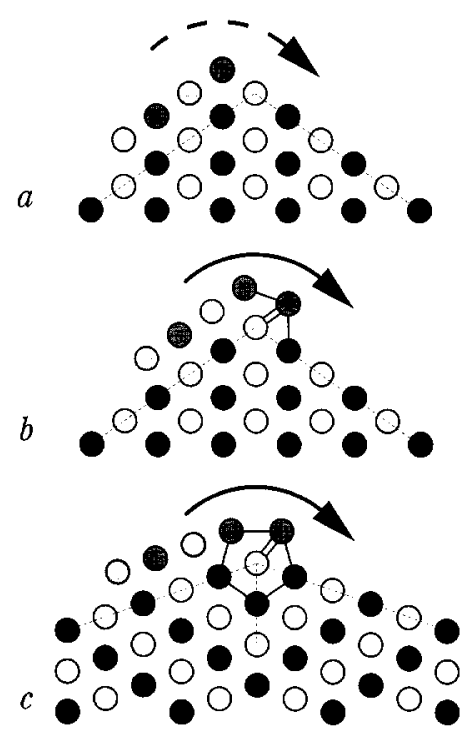

Fig. 4. Crystal-defects and "growth around the corner". (a) A correctly deposited layer (shaded) on a perfect crystal cannot proceed by stepflow over the [101] edge between $\{111\}$ faces; it merely extends the surface of the adjoining (111) face. The same applies to (111)/(100) edges. (b) An incorrectly deposited layer (i.e. in stacking fault position) can proceed over the edge, since a $141^{\circ}$ reentrant corner is created. In passing the edge, the registry of the new layer switches from "wrong" (hcp) to "right" ( $f(c)$ ). Apparently, the same process can not proceed in the opposite direction (a). (c) An incorrectly deposited layer can proceed over a twin-ridge, as can a correctly deposited layer (Fig. 3d). This process results in local fivefold symmetry, as indicated by the pentagon.

single crystal is only possible when the advancing layer is in hcp stacking fault position (Figs. 4a and 4b). A special case is shown in Fig. 4c, that illustrates that the ridge of Fig. 3 can also be overcome when the advancing layer is in hcp stacking fault position, although the coordination of the nucleation site is somewhat less than fourfold, owing to the length of the horizontal bond in the highlighted pentagon, which is 1.09 , rather than $1.00^{3}$.

\section{Cross-twinning model}

As pointed out in Section 2, the WHS-model allows for accelerated growth in only two dimen-

\footnotetext{
${ }^{3}$ Corresponding to a $16 \%$ loss in $\mathrm{LJ}$ binding energy.
} 

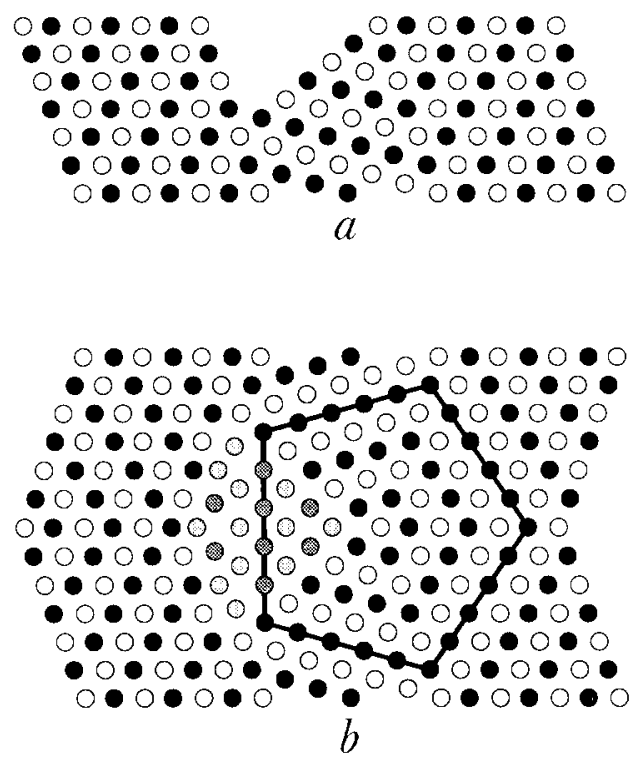

Fig. 5. Construction of a possible atomic configuration in the cross-twinning region. (a) As Fig. 3a, but with the L-part extended to make the upper layers of $L$ and $U$ continuous. (b) Reflection twinning on this upper layer produces an empty region that has nearly the dimensions of a sector in a pentagon. Completing this pentagon as shown (shaded atoms) results in an incoherent grain boundary with both L-parts. The structure now contains the crossing of a twin lamella (two parallel twin planes) with a third twin plane. Repeating this procedure results in Fig. 6.

sions, when an otherwise perfect fcc crystal contains at least one twin lamella. In the previous section it was demonstrated that this type of growth resists stacking faults. However, as is clear from Fig. $2 \mathrm{~g}$, this type of growth results in thin hexagonal platelets with well developed (111) faces, as is actually observed. Clearly, the reason for this is the absence of grooves and ridges on the (111) faces, and the remedy is obvious: introduce a second lamella in the crystal that intersects these (111) faces. Clearly, this lamella cannot be parallel to the first, but must be parallel to one of the $\{\overline{1} 11\}$ faces, e.g. parallel to the (111) surfaces of the L- and U-parts of Fig. 3a, and will cross the first lamella. In order to see whether this is possible, and to examine the local atomic arrangement in the crossing region, two new twin planes (that sandwich the second lamella) must be introduced in Fig. 3a. This is achieved in two steps: Fig. 5 shows the effect of introducing a single (horizontal) twin plane in the structure of Fig. 3a: in
Fig. 5a the L-part of Fig. 3a is extended to make its surface continuous with that of the U-part (this is only possible when the number of layers in the first lamella, the M-part, is a multiple of 3); subsequently, Fig. 5a is mirrored across its uppermost line of atoms, to leave a kite-like open space. This space can be filled by mirroring the atoms in the lamella across the surface of the lamella, Fig. 5b. The latter atoms do not fill the space exactly: there is some mismatch with the domains to the left, and the emerging pentagonal arrangement (highlighted by bold lines) consists of four undistorted fec sectors, with top-angles of $70.5^{\circ}$, and one strained sector, with top-angle $77.9^{\circ}$. The second step in the construction is to introduce a second horizontal twinplane in the structure of Fig. 5b, to complete the horizontal lamella. This can be done in the same way

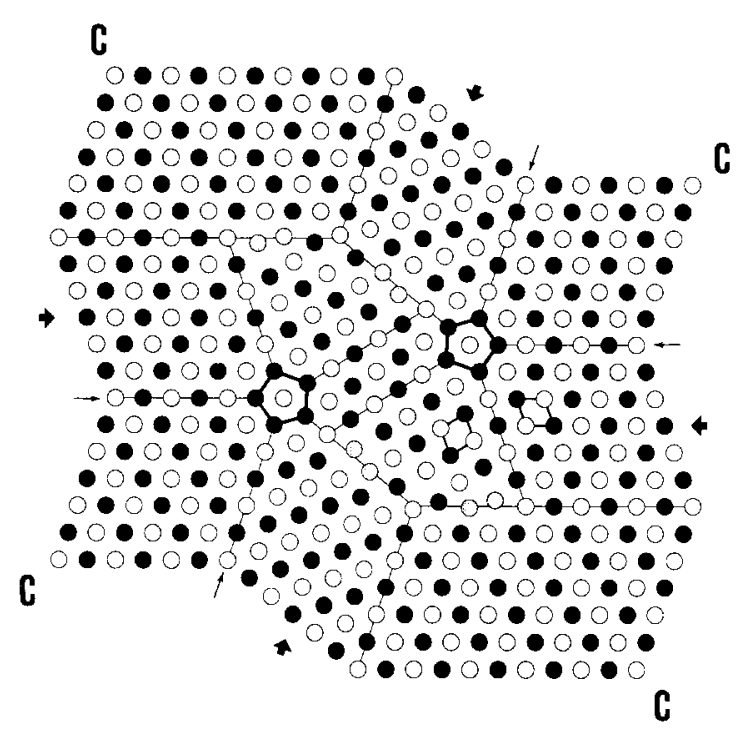

Fig. 6. Possible atomic arrangement in the crossing region of two twin lamellae (projection and black and white shading of circles as in Fig. 3; light shading denotes uncertain positions at incoherent domain boundaries). A large single crystal (to which the domains marked " $\mathrm{C}$ " belong) contains two crossing twin lamellae (each indicated by two bold arrows; thin arrows mark reentrant corners). There are two axes of approximate 5-fold rotation symmetry (highlighted by bold pentagons), each axis being surrounded by 4 undistorted and 1 slightly distorted $f c c$ domains. The distortion can be seen by comparing similar rhombs in both types of domain. The ratio of rhomb-diagonals in the distorted domains is 1.24 , rather than $1.41(\sqrt{2})$, as has been actually observed in fcc crystals of $\mathrm{C}_{60}$. (If the drawing is rotated some $70^{\circ}$ in a clockwise direction, its lower part corresponds to Fig. 5b.) 
as with the first plane, and will produce a second (approximate) fivefold axis in the crystal. The final result is shown in Fig. 6. The structure is seen to have reentrant corners (thin arrows) on all closepacked faces (including those not perpendicular to the page), which will now stimulate fcc ABC stacking in three, rather than in two dimensions.

It should be emphasized here that the cross-twinning as described is only possible in an fcc crystal, because there are 4 non-parallel planes on which mirror twinning is possible. Conversely, cross-twinning in a hcp crystal is not possible because there is only one close-packed twin plane. Since these special properties of the fcc crystal are used in the twinning construction, cross-twinning by itself cannot be the single reason for the preference of $\mathrm{fcc}$ over hcp of many $\mathrm{vdW}$ crystals. However, rather than viewing the, essentially non-crystalline, atomic arrangement in the crossing region as a result of cross-twinning, it might be the origin of the crystal, in line with what is known about structure in the early stages of crystallization.
Small particles are known to adopt fivefold symmetry very easily. In 1952 it was pointed out by Frank [9] that, since the average coordination in the liquid is close to 12 , the 12 atoms in the first coordination shell of a particular atom are more likely to be arranged at the corners of an icosahedron, than at the corners of an ( $\mathrm{fcc}$ ) cuboctahedron or an (hcp) anti-cuboctahedron, when the liquid is supercooled. As noted already in the introduction, the same conclusion is reached by applying atom-byatom aufbau-algorithms [5]. Today many substances are known that "crystallize" initially into multiply twinned particles (MTPs), frequently in the form of icosahedra or pentagonal bipyramids (decahedra). With reference to Fig. 6, however, it is clear that no complete icosahedra are present in the structure. Rather, the highlighted pentagons denote stacks of pentagons, perpendicular to the drawing, with all pentagons in the same orientation. Multishell icosahedra (MICs) contain such stacks. Thus, the atomic arrangement around each of the fivefold axes in Fig. 6 is the same as that in a multishell decahedron, that

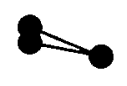

$a$

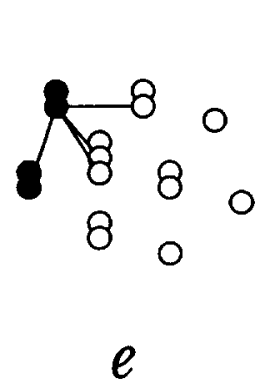

$e$

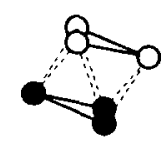

$b$

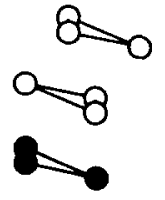

c

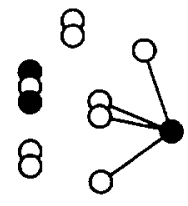

$d$
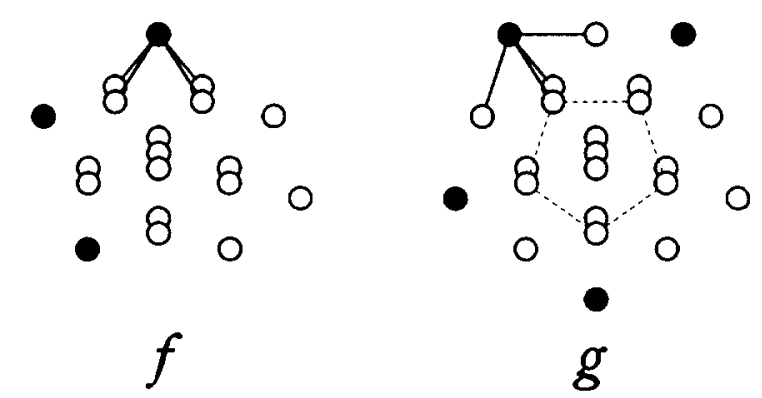

Fig. 7. A possible aufbau-sequence for a 23 -atom pentagonal decahedron, involving the coalescence of trimers. The last atoms added are in black. Atoms added in one picture are symmetry equivalent. The new bonds made by one of these atoms are indicated in (d)-(g). (a) and (b) Coalescence of two parallel trimers in a star-of-David configuration produces an octahedron. (c) A third trimer results in two octahedra sharing a face. (d) Occupation of three reentrant corners. (e) Completion of two pentagonal rings (the first of these 4 equivalent atoms can make only three bonds; a dimer would make 6 bonds). (f) and (g) Occupation of remaining 4-coordinated sites. The view is approximately down the fivefold axis. 
may be thought of as taken out from a MIC. A MIC cannot be modelled through atom-by-atom addition to a single shell icosahedron; in fact, the growth of (observed) MICs is still unexplained ${ }^{4}$. The origin of decahedra can be understood from an aufbau-algorithm that allows addition, not only of single atoms, but also of dimers, triangular trimers, or tetrahedral tetramers, that will be abundant in the supercooled liquid. Fig. 7 shows a possible growth scenario for a 23 -atom pentagonal decahedron, that starts with the coalescence of 3 parallel but staggered trimers (two such trimers form an octahedron), and continues with atom-by-atom addition to the most favourable sites, in the same way as the 13-atom icosahedron can be built. Fig. 6 suggests that a fortuitous coalescence or intergrowth of two such pentagonal nuclei is needed to trigger fcc growth.

A less complicated arrangement can be obtained when the construction is based on Fig. 3e. Fig. 8 shows the atomic arrangement in the crossing region of two non-parallel stacking faults. This simplified model has been proposed earlier to explain fcc crystal growth [11], but appears to be less appropriate in view of experimental evidence (Section 5).

Growth on a support does not require fault crossing at all, since two dimensions of the crystal to grow are determined by the lateral extensions of the first layer to be deposited on the support. Although the support is supposed here to be featureless to the extent that it does not prevent the first layer to adopt a close-packing arrangement in two dimensions, it may contain steps whose heights do not match the vertical layer-to-layer distance $d_{111}$ of the deposit (Fig. 9a). The stepheight may, however, be close to $\frac{1}{3} d_{111}$ or $\frac{2}{3} d_{111}$ of the deposit. Fig. 9b depicts a situation where an island has been nucleated on top of the first layer, with a step-edge proceeding in the direction of the supportstep. Eventually the rearrangement of Fig. 9c may occur, resulting in a

\footnotetext{
${ }^{4}$ Rather than growing "outward", by acquiring new shells, an "empty" MIC, i.e. a MIC consisting only of the outer shell, surrounding a liquid droplet, could more easily grow inward by virtue of the reentrant corners present on the inner surface of the outer shell. Results of MD-simulation of surface freezing of liquid droplets [10] are suggestive in this direction.
}

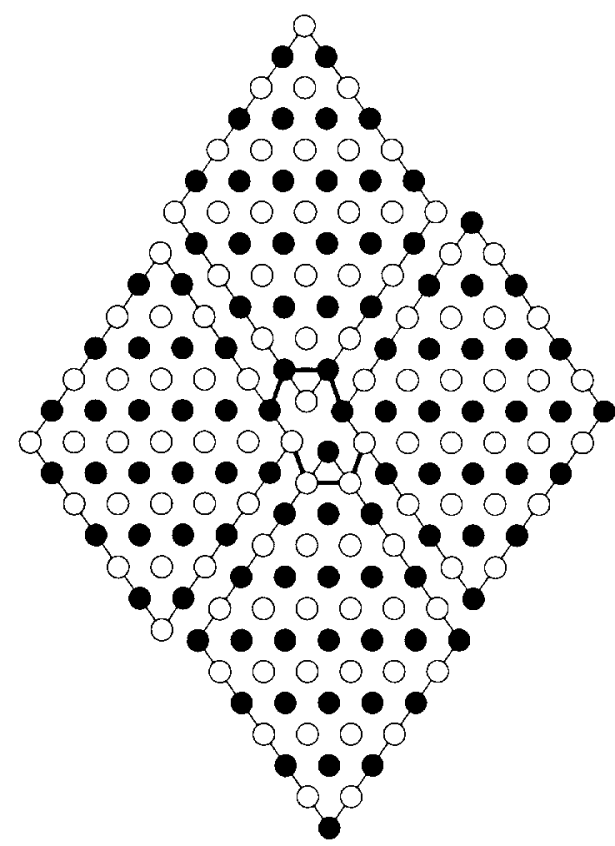

Fig. 8. Crossing of single stacking faults. The crossing consists of two stacks (perpendicular to the page) of incomplete pentagons, as indicated by bold lines. The incomplete matching in the centre leaves a vacancy channel surrounded by atoms with 11 , rather than 12 nearest neighbours. All faces exhibit steps of height $\frac{1}{3} d_{111}$ (like colours) or $\frac{2}{3} d_{111}$ (unlike colours).

surface structure with the same properties as that of Fig. 3e.

\section{Experimental evidence}

If, for a particular substance, e.g. Ar, the growth model outlined in the preceding section is indeed the reason for its preference for the fcc crystal structure over all other close packings, no crystal, however small, should be found without cross-twinning. This can be verified by (electron-) diffraction experiments on small particles or clusters. Thus Farges et al. $[2,12]$ have obtained diffraction patterns for Ar-clusters, produced by adiabatic expansion in supersonic beams, for a variety of sizes, ranging from a few tens to some thousands of atoms. From these experiments it has become clear that the largest clusters observed are fcc, but that fivefold symmetry, in the form of MICs or polyicosahedral arrangements, has to be incorporated in simulation models to match the ob- 

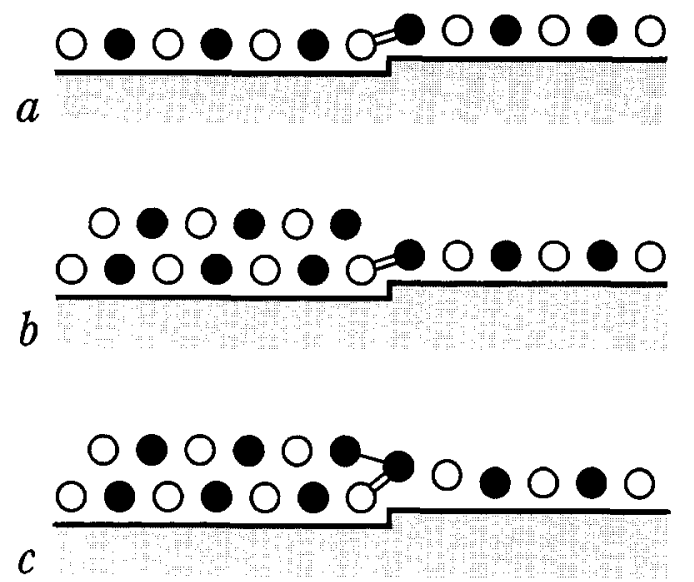

Fig. 9. Growth on a substrate may induce fcc stacking. (a) A close packed layer covers a support with its close packed rows aligned with one of the steps that are supposed to be present on the support face, but that do not match the layer distance $d_{111}$ of the deposit. The steps may be a fraction of $d_{11}$, or a multiple plus a fraction. In the latter case the multiple is supposed to be occupied by a stack of layers of the deposit. (b) The edge of a second layer advances to the right until it reaches the step in the first layer. (c) The first row of atoms at the higher side of the step can now be drawn in the more favourable position between the two layers at the left, thus producing a $\frac{1}{3} d_{11}$ step with a row of 4-coordinated sites that stimulate further fcc growth. The situation is essentially the same as that of Fig. 3e.

served diffraction patterns, the more so as the clusters are smaller. Although clusters of different sizes are produced in different experiments, a plausible scenario is to assume the smaller clusters to be the structural precursors of the larger clusters. In the model, developed in the preceding section (Fig. 6), there is a limited region where fivefold symmetry is dominant, surrounded by a region that is mainly single crystalline; only the latter part (i.e. the fcc crystalline contribution to the diffraction patterns) increases with clusterising, making deviations from the ideal fcc pattern disappear gradually. According to this view there is no structural transition from non-crystalline (fivefold) to crystalline symmetry, only a smoothly decreasing share of the non-crystalline part. Diffraction patterns have been calculated for the simple crossing stacking fault model [13]. They are significantly different from patterns of perfect fcc crystals (as are the observed traces, even for clusters of some 3000 atoms), but compare reasonably well with the experimental results for $500<N$
$<3000$. However, it was found that at least 4 crossings were necessary in the models to obtain best agreement. This may be due to the fact that the regions with local fivefold symmetry are small, as a result of the fact that the crossing twin-lamellae consist of only a single layer (cf. Fig. 8). Starting with the arrangement of Fig. 6, with six layers in the crossing lamellae, a 3343-atom structural model for an octahedral Ar-cluster was constructed, and relaxed under a LJ-potential, cf. Fig. 10. The calculated diffraction pattern $\left(s^{3} I(s)\right.$, rather than $I(s)$; cf. Ref. [13] for calculation details), Fig. 11b, is in striking agreement with the observed pattern [2], Fig. $11 \mathrm{a}$ [14]. This is even more remarkable when traces a and $b$ are compared with those of other models, e.g. perfect fcc (Fig. 11c), multishell icosahedral (Fig. 11d), and decahedral (Fig. 11e). Only in the case of the icosahedral structure the splitting of the first peak is unresolved, as observed; however, beyond $s \approx 6$ $\AA^{-1}$ significant line broadening, presumably resulting from strains in the outer shells, is not observed. This suggests that the combination of two nuclei with fivefold symmetry, as in Fig. 10, helps to reduce the strains (a close inspection of Fig. 10 reveals that the relaxation procedure has resulted in an extra stacking-fault, to the effect that the total surface of incoherent grain-boundaries is reduced).

Even if a convincing explanation was available for the crystal structure of the heavier rare gases, based on an atomic interaction model, this would be of little help to explain the fcc crystal structure of

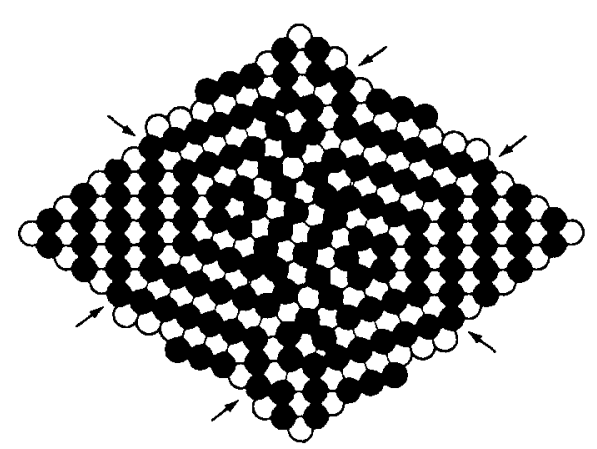

Fig. 10. [110] cross-section of an LJ-relaxed 3343-atom octahedral fragment of the structure of Fig. 6. Arrows mark atomic-scale reentrant corners, that act as preferred surface nucleation sites. The two extra sites (as compared to Fig. 6) result from the relaxation procedure. 


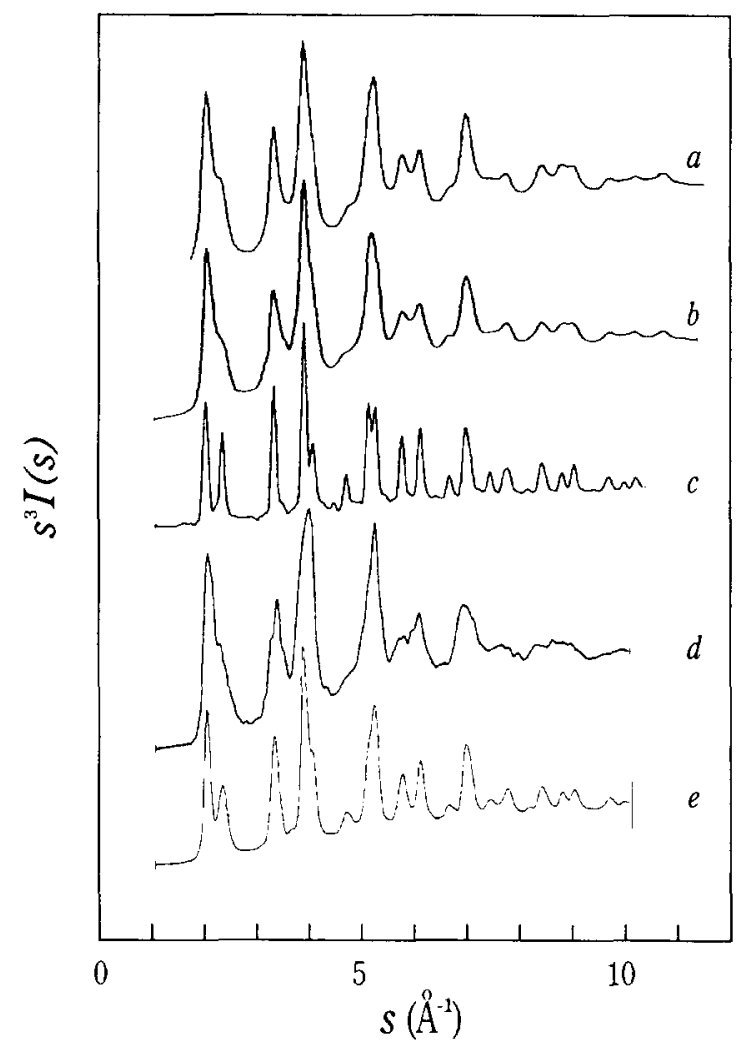

Fig. 11. Simulated electron-diffraction pattern (b) of the structure of Fig. 10, compared with the experimentally observed pattern (a) for $N \approx 3000$ atom Ar clusters [2]. Other simulated patterns are included for comparison: 3281-atom fcc cluster (c), 2869-atom multishell icosahedral cluster (d), and 2622-atom decahedral cluster (e).

$\mathrm{C}_{60}{ }^{5}$. The large inter-layer distances involved make it improbable that the observed crystal structure is a result of long-range interactions (other than structural information that may be transferred by a static molecule from the layer below to the layer above, like in LT C $_{70}$ [16]). Conversely, an explanation for $\mathrm{C}_{60}$ could also be applicable to the heavier rare

\footnotetext{
${ }^{5}$ Both $C_{60}$ and the heavier rare gases are known to have hcp phases as well, c.f. Ref. [15]. The origin may be in the extreme sensitivity of stacking order to small amounts of impurity molecules, notably $\mathrm{O}_{2}, \mathrm{~N}_{2}$ and $\mathrm{CO}$ in the case of the rare gases and, possibly, $\mathrm{C}_{70}$ in the case of $\mathrm{C}_{60}$. Non-integral surface steps could be decorated by these molecules, modifying the step structure that underlies the proposed growth model.
}

gases. The explanation, that is proposed here, relies on the modification of the crystal surface through its intersection with internal defects. Surface features on crystalline $C_{60}$ can now be observed with molecular resolution by atomic force microscopy. Some puzzling features, that were found on some faces, fit well into the picture of Fig. 6. Although local fivefold symmetry is not directly observed, the frequent occurrence [17] of the angle $72^{\circ}$ is suggestive in that direction. Also, the ratio of rhomb-diagonals, 1.24 (rather than $\sqrt{2}=1.41$ ) in the distorted regions of Fig. 6, exactly reproduces the observed value [18].

$\mathrm{C}_{60}$ crystals with only parallel twin planes (i.e. no crossing) have been observed to be thin in a direction perpendicular to the twin planes (i.e. fast growing in the in-plane directions $\langle\overline{1} 11\rangle$ and $\langle 1 \overline{1} \overline{1}\rangle$, but slow-growing in the $\langle 111\rangle$ directions). Diffraction patterns of such platelets reveal diffuse streaking in the [111] direction, but not in the [111] and [111], etc., directions, consistent with the picture of fcc stacking order in all directions, except in the [1111] direction. The puzzling phenomenon of stacking disorder in only one out of four equivalent directions is now explained by the absence of grooves and ridges on the (111) faces [19].

Icosahedral clusters of $\mathrm{C}_{60}$ molecules have been observed [20], and icosahedral $\left(\mathrm{C}_{60}\right)_{13}$ and $\left(\mathrm{C}_{60}\right)_{55}$ have been predicted to be stable on the basis of molecular dynamics simulations [21]. Similarly, (benzene) ${ }_{13}$ has been predicted [22] and observed [23] to be icosahedral, although $\mathrm{C}_{6} \mathrm{H}_{6}$ is obviously less spherical. This fact could be of importance in giving an explanation for the (distorted) fcc crystal structure of benzene in the spirit of the proposed model, rather than by performing lattice energy calculations on infinite crystals in the observed spacegroup.

Although the proposed growth model presupposes weak short range isotropic interatomic or intermolecular interactions, it is of interest to consider the crystallization behaviour of some fcc transition metals, in particular those, for which the calculated difference between fcc and hcp stacking is small, i.e. the noble metals [24]. Thus, $\mathrm{Cu}, \mathrm{Ag}$ and $\mathrm{Au}$, and also $\mathrm{Ni}, \mathrm{Pd}$ and $\mathrm{Pt}$, are known to form small particles exhibiting fivefold symmetry (MTPs) [25]. This may be simply the result of the small hcp/fcc difference, predicted by local density functional theory, but could also play a role in the final choice for the fcc 
structure, along the lines described above. Gold and platinum crystals have been observed to grow according to the WHS-scheme [26]. A complicating factor in connecting the preference for fcc crystallization of gold to this behaviour is the well-known $\mathrm{fcc} / \mathrm{hcp}$ herringbone surface reconstruction. It is well-known that the crystalstructure of gold is predicted wrongly: bcc [24]; Cobalt is predicted to be fcc. It should be borne in mind that these predictions are based on infinite-crystal calculations, at the observed density, and that the fcc/hcp energy differences are below the $5 \%$ level, where the accuracy of the cohesive energy is only $25 \%$; nevertheless, the correct prediction of the hcp-bcc-hcp-fcc trend in the transition metals is remarkable.

Strong evidence would be expected to come from hetero-epitaxy experiments, where a hcp material is deposited on the (111) face of an fcc material with negligible lattice mismatch, i.e. when the hcp material was forced by the support to adopt the fcc structure. This is indeed the case for $\mathrm{Co} / \mathrm{Cu}(111)$, but the phenomenon is believed to originate mainly in the possibility to reduce lattice mismatch to $1.8 \%$ for $\mathrm{fcc} / \mathrm{fcc}$, as compared to $2.0 \%$ for $\mathrm{hcp} / \mathrm{fcc}$, for the $\mathrm{Co} / \mathrm{Cu}$ interface [27].

Unexplained preference for fcc over hcp is also found on a quite different scale, i.e. $0.1-1 \mu \mathrm{m}$, in sediments of synthetic (latex) or natural (silica) spheres from suspensions with a narrow sphere-size distribution. Thus, electron microscopy studies of cleaved opals reveal a (frequently faulted) fcc structure; although steps like that of Fig. 9 were observed, the clear preference for fcc was, tentatively, ascribed to (non-observed) screw-dislocation spiralgrowth [28]. However, if the structure had been hcp this could have been explained in the same way.

Finally, but possibly most important, is the success of the WHS-theory to explain the observed growth-forms of germanium; although not meant to explain the observed crystal structure, the importance of grooves on the surface as surface-nucleation centres was convincingly demonstrated.

From the data, given by Hamilton and Seidensticker, it can be estimated that an area of some 5000 $\AA^{2}$ can be covered through nucleation in a single groove, per unit length $(\AA)$ of the groove, so parallel grooves could be as far apart as $5000 \AA$ to prevent wrong stacking on an extended face (in $\mathrm{Ge}$ ). A rough estimate, assuming the same number of atoms between successive grooves, gives an upper limit of $10^{10}$ atoms in an fcc argon crystal, grown from a single non-crystalline nucleus, before new stacking faults (and, consequently, new grooves) occur.

\section{Discussion}

An atomic-scale growth-model for vdW crystals with effectively spherical molecules has been presented which favours fcc over hcp. This preference is explained as being closely connected with kinetic effects that dominate surface nucleation, that is, the observed crystal structure is not necessarily that of lowest free energy of the infinite crystal, but corresponds to a minimum that can be reached by a growth process, i.e. by a sequence of events and aggregates of increasing size, whose members are not separated by high energy barriers, are not structurally instable, and cannot all correspond to the global minimum for their size. A crucial role is played by particles with non-crystalline, fivefold symmetry, in particular by decahedra, but possibly also by icosahedra. These particles are known to be stable and preferred in a limited size domain by fcc materials whose hypothetical hcp counterparts can be expected to have virtually the same binding energy. A long-standing problem, viz. how does the pentagonal particle manage to transform into a crystalline particle when its size exceeds the crossing point where crystalline arrangements become energetically more favourable than non-crystalline arrangements, seems to be removed by the proposed growth-model: rather than opposed to, and frustrating crystal growth, pentagonal particles seem to be essential in the early stages, in particular to lower the barriers for surface nucleation. According to this model, the pentagonal particles remain virtually unchanged in the interior of the growing crystal, i.e. no structural transition is needed.

Evidence is mainly based on the observed $[6,7,19,26]$ and simulated $[8,29]$ effect of surface grooves on the rate of surface nucleation and growth, and on the observation [11] that this type of nucleation preserves the stacking-order. The requirement to have such grooves on all close-packed faces (resulting in the cross-twinning model) is a direct 
consequence of extending the essentially two-dimensional observed growth effects into three dimensions.

The dominance of pentagonal arrangements in small Ar-clusters has been convincingly demonstrated by electron-diffraction experiments $[2,12]$. Nevertheless large Ar-clusters, produced by the same technique, are unmistakably fcc, albeit that vestiges of fivefold symmetry can be inferred from deviations of the experimental patterns from simulations, based on perfect fcc crystals. It should be remembered, that the clusters are produced under non-equilibrium conditions, and do not necessarily mimic the evolution of structure in a freezing liquid.

As noted already, simulations of freezing invariably result in mixed fcc/hcp stackings [30], although pentagonal arrangements are frequently observed in the early stages [31]. The extent to which these simulations are realistic is unclear; reported "crystallizations" occur in the whole simulation volume at a time, i.e. surface effects on a localized nucleus, as believed to be essential in the present model, do not play a role; the influence of the application of periodic boundary conditions has been the subject of debate [32]. From Fig. 6 it is clear that a rather complicated structure may be required in addition to thermodynamic constraints to trigger the crystallization process; such a structure cannot be expected to occur "spontaneously" in a simulation within an acceptable time (the liquid could be "seeded", however).

Surface effects are modelled in simulations of the liquid-solid interface (which obviously requires the assumption of a crystal structure). However, the results are virtually the same, as far as the stacking order is concerned [33]; apparent exceptions [34] may occur by chance, especially when the number of layers considered is small. It is possible to introduce in these simulations stackingorder preserving surface features, like a groove. Simulations with a nonintegral step (like that of Fig. 3e) have been unsuccessful, however [35].

Essential to the proposed model is that surface nucleation will take place almost exclusively in fourfold coordinated sites, in agreement with the assumption of an octahedral equilibrium shape of a single crystal, in which only the slow growing faces are exposed. Indeed, at $T \approx 0.4 T_{\mathrm{m}}$, a 4-coordinated site's chance to be occupied is nearly 60 times the chance for a 3-coordinated site, for an LJ-crystal, but the number of 4-coordinated sites in the reentrant corners is only a small fraction of all surface sites. Moreover, many fcc crystals, notably $\mathrm{C}_{60}$ [36], exhibit $\{100\}$ faces as well, suggesting that not all 4-coordinated sites will be occupied. Presumably, it is not only the 4-coordination of the type- 3 sites, but also the larger scale geometry of the grooves, that makes the grooves preferable as nucleation sites. Thus, the grooves are believed to be acting as obstacles to surface diffusion, significantly increasing the average local residence time of an atom, which in addition to the local fourfold coordination, makes the groove an effective sink. Such sinks are not present on plane faces, in particular (100).

The fcc/hcp dilemma is best known and most pronounced in connection with the rare gases, and with MD-simulations of crystal nucleation and growth in simple liquids. It will be of interest, however, to investigate whether the observed spacegroup of molecular crystals of, e.g., $\mathrm{CCl}_{4}, \mathrm{CO}_{2}, \mathrm{SF}_{6}$, $\mathrm{Cl}_{2}$, etc, is actually the best choice, as far as the cohesive energy is concerned, or that a hcp stacking would be equally or even more favourable. Even in the case of $\mathrm{C}_{60}$, such an investigation has not yet been performed.

\section{References}

[1] D.A. Young, Phase Diagrams of the Elements (University of California Press, Berkeley, 1991).

[2] J. Farges, M.F. de Feraudy, B. Raoult and G. Torchet, J. Chem. Phys. 84 (1986) 3491; Adv. Chem. Phys. 70 (1988) 45.

[3] B.W. van de Waal, J. Chem. Phys. 90 (1989) 3407; J. Xie, J.A. Northby, D.L. Freeman and J.D. Doll, J. Chem. Phys. 91 (1989) 612; B. Raoult, J. Farges, M.F. de Feraudy and G. Torchet, Phil. Mag. B 60 (1989) 881.

[4] J.J. Burton, J. Chem. Phys. 52 (1970) 345; 56 (1972) 3133; Chem. Phys. Lett. 7 (1970) 567; 17 (1972) 199; J. Chem. Soc. Faraday Trans. II 69 (1973) 540; Nature (London) 229 (1971) 335; Acta Met. 21 (1973) 1225; M.R. Hoare and P. Pal, Nature (London) 230 (1971) 5; 236 (1972) 35; J. Crystal Growth 17 (1972) 77; Adv. Phys. 20 (1971) 161; 24 (1975) 645

[5] M.R. Hoare, Adv. Chem. Phys. 40 (1979) 49.

[6] R.S. Wagner, Acta Met. 8 (1960) 57.

[7] D.R. Hamilton and R.G. Seidensticker, J. Appl. Phys. 31 (1960) 1165 .

[8] N.-B. Ming, K. Tsukamoto, I. Sunagawa and A.A. Chernov, 
J. Crystal Growth 91 (1988) 11; J.-M. Jin, N.-B. Ming and A.A. Chernov, J. Crystal Growth 98 (1989) 341.

[9] F.C. Frank, Proc. Roy. Soc. (London) Ser. A 215 (1952) 43.

[10] H.S. Lim, C.K. Ong and F. Ercolessi, Comp. Mat. Sci. 2 (1994) 495.

[11] B.W. van de Waal, Phys. Rev. Lett. 67 (1991) 3263.

[12] J. Farges, M.F. de Feraudy, B. Raoult and G. Torchet, J. Chem. Phys. 78 (1983) 5067.

[13] B.W. van de Waal, J. Chem. Phys. 98 (1993) 4909.

[14] These results were first reported at the ICCG-XI conference, The Hague (1995).

[15] L. Meyer, Advan. Chem. Phys. 16 (1969) 343; J.L. de Boer, S. van Smaalen, V. Petricek, M. Dusek, M.A. Verheijen and G. Meijer, Chem. Phys. Lett. 219 (1994) 469.

[16] Y. Guo, N. Karasawa and W.A. Goddard III, Nature 351 (1991) 464.

[17] B. Keita, L. Nadjo, R. Céolin, V. Agafonov, D. André, H. Szwarc, J. Dugué, C. Fabre and A. Rassat, Chem. Phys. 179 (1994) 595.

[18] B.W. van de Waal, Chem. Phys. 186 (1994) 447.

[19] P.J.F. Harris, R.E. Douthwaite, A.H.H. Stephens and J.F.C. Turner, Chem. Phys. Lett. 199 (1992) 631; B.W. van de Waal, Chem. Phys. Lett. 202 (1993) 341.

[20] Y. Saito, Y. Ishikawa, A. Ohshita, H. Shinohara and H. Nagashima, Phys. Rev. B 46 (1992) 1846; T.P. Martin, U. Näher, H. Schaber and U. Zimmermann, Phys. Rev. Lett. 70 (1993) 3079.

[21] D.J. Wales, J. Chem. Soc. Faraday Trans. 90 (1994) 1061; J. Chem. Phys. 101 (1994) 3750.
[22] B.W. van de Waal, J. Chem. Phys. 79 (1983) 3948.

[23] D.C. Easter, R.L. Whetten and J.E. Wessel, J. Chem. Phys. 94 (1991) 3347; S.M. Beck and J.H. Hecht, J. Chem. Phys. 96 (1992) 1975; D.C. Easter, J.T. Khoury and R.L. Whetten, J. Chem. Phys. 97 (1992) 1675; D.C. Easter, A.P. Baronavsky and M. Hawley, J. Chem. Phys. 99 (1993) 4942.

[24] H.L. Skriver, Phys. Rev. B 31 (1985) 1909; D.G. Pettifor, Mater. Sci. Technol. 1 (1993) 61.

[25] See: Faraday Disc. 92 (1991) 1-300.

[26] R.H. Morriss, W.R. Bottoms and R.G. Peacock, J. Appl. Phys. 39 (1968) 3016; P.J.F. Harris, J. Catal. 97 (1986) 527.

[27] P. Bödeker, A. Abromeit, K. Bröhl, P. Sonntag, N. Metoki and H. Zabel, Phys. Rev. B 47 (1993) 2353.

[28] E.A. Monroe, D.B. Sass and S.H. Cole, Acta Cryst. A 25 (1969) 578.

[29] H. Li, X.-D. Peng and N.-B. Ming, J. Crystal Growth 139 (1994) 129.

[30] S. Nosé and F. Yonezawa, J. Chem. Phys. 84 (1986) 1803.

[31] H. Jónsson and H.C. Andersen, Phys. Rev. Lett. 60 (1988) 2295; W.C. Swope and H.C. Andersen, Phys. Rev. B 41 (1990) 7042 .

[32] J.D. Honeycutt and H.C. Andersen, Chem. Phys. Lett. 108 (1984) 535; J. Phys. Chem. 90 (1986) 1585.

[33] E. Burke, J.Q. Broughton and G.H. Gilmer, J. Chem. Phys. 89 (1988) 1030.

[34] J.P. van der Eerden, Faraday Disc. 95 (1993) 65.

[35] J.Q. Broughton, private communication.

[36] M.A. Verheijen, H. Meekes, G. Meijer, E. Raas and P. Bennema, Chem. Phys. Lett. 191 (1992) 339. 